ARTICLE

\title{
Alterations of the human gut microbiome in multiple sclerosis
}

Sushrut Jangi ${ }^{1, \star}$, Roopali Gandhi ${ }^{1}{ }^{\star \star}$, Laura M. Cox ${ }^{1}$, Ning Li $^{2}$, Felipe von Glehn ${ }^{1}$, Raymond Yan ${ }^{1}$, Bonny Patel ${ }^{1}$,

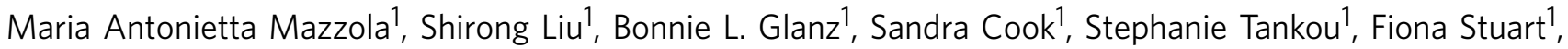
Kirsy Melo ${ }^{1}$, Parham Nejad ${ }^{1}$, Kathleen Smith¹, Begüm D. Topçuolu ${ }^{3}$, James Holden ${ }^{3}$, Pia Kivisäkk ${ }^{1}$, Tanuja Chitnis ${ }^{1}$, Philip L. De Jager', Francisco J. Quintana', Georg K. Gerber², Lynn Bry² \& Howard L. Weiner ${ }^{1}$

The gut microbiome plays an important role in immune function and has been implicated in several autoimmune disorders. Here we use 16S rRNA sequencing to investigate the gut microbiome in subjects with multiple sclerosis (MS, $n=60)$ and healthy controls $(n=43)$. Microbiome alterations in MS include increases in Methanobrevibacter and Akkermansia and decreases in Butyricimonas, and correlate with variations in the expression of genes involved in dendritic cell maturation, interferon signalling and NF-kB signalling pathways in circulating T cells and monocytes. Patients on disease-modifying treatment show increased abundances of Prevotella and Sutterella, and decreased Sarcina, compared with untreated patients. MS patients of a second cohort show elevated breath methane compared with controls, consistent with our observation of increased gut Methanobrevibacter in MS in the first cohort. Further study is required to assess whether the observed alterations in the gut microbiome play a role in, or are a consequence of, MS pathogenesis.

\footnotetext{
${ }^{1}$ Ann Romney Center for Neurologic Diseases, Evergrande Center for Immunologic Diseases, Partners Multiple Sclerosis Center, Brigham and Women's Hospital, Department of Neurology, Harvard Medical School, Boston, Massachusetts 02115, USA. ${ }^{2}$ Center for Clinical and Translational Metagenomics, Department of Pathology, Brigham and Women's Hospital, Harvard Medical School, Boston, Massachusetts 02115, USA. ${ }^{3}$ Department of Microbiology, University of Massachusetts, Amherst, Massachusetts 01003, USA. * These authors contributed equally to this work. Correspondence and requests for materials should be addressed to H.L.W. (email: hweiner@partners.org).
} 
$\mathrm{M}$ icroorganisms in the human gut encompass hundreds to thousands of bacterial, archaeal, viral and fungal species, making the human intestinal lumen a rich and dense source of antigenic diversity ${ }^{1}$. The gut mucosal immune system samples and processes these microbial antigens, potentially driving the expansion of particular immune subsets or generating specific immune repertoires ${ }^{2}$. Thus, the intestinal microbiome is an important entity within the host that influences immune responses both locally and systemically.

The gut microbiome has been implicated in numerous immunologic disorders, including multiple sclerosis (MS), inflammatory bowel disease, type 1 diabetes and rheumatoid arthritis $^{3-5}$. In experimental autoimmune encephalomyelitis (EAE), a murine model for MS, altering the gut microbiome modulates central nervous system (CNS) autoimmunity. In a relapsing-remitting mouse model of spontaneous EAE, transgenic SJL/J mice raised in germ-free conditions were protected against developing the disease, while the introduction of commensal microbiota into the gut restored susceptibility ${ }^{6}$. While gnotobiotic mice are relatively immunocompromised due to lack of microbial stimulation promoting immune maturation, specific association of germ-free mice with defined commensal species has been shown to modulate the development and severity of EAE. Segmented-filamentous bacteria (SFB) drive expansion of Th17 cell populations and generation of interleukin (IL)-17 in the gut $^{7}$. Mono-colonization of the gut of C57BL/6 mice with segmented-filamentous bacteria promotes Th17 accumulation in the spinal cords of mice and induces the development of EAE ${ }^{8}$. Conversely, treatment of C57BL/6 mice with a polysaccharide from the organism Bacteroides fragilis expands intestinal Foxp3 + CD4 Tregs and protects against the development of CNS autoimmunity 9,10 .

In the case of human autoimmune disease, associations have been reported with different members of the commensal microbiota. In a study of 20 MS patients versus 40 healthy controls, Faecalibacterium, Prevotella and Anaerostipes were decreased in MS, but the connection between microbiota, treatment and changes in immunity was not examined ${ }^{11}$. Prevotella copri has been associated in proinflammatory conditions, and has found to be enriched in patients with new-onset rheumatoid arthritis ${ }^{5}$, or capable of exacerbating dextran sodium sulfate colitis in antibiotic-treated C57BL/6 mice. Butyrate-producing organisms have protective associations with inflammatory conditions, for example, Faecalibacterium prausnitzii has been shown to be reduced in inflammatory bowel disease ${ }^{12}$. In neuromyelitis optica, a CNS autoimmune disease directed against aquaporin-4, there are increased antibodies against gastrointestinal antigens and cross-reactivity to a protein belonging to Clostridium perfringens, suggesting that autoimmunity in neuromyelitis optica may be driven by molecular mimicry against microbial antigens ${ }^{13}$. Similarly, the autoimmunity associated with Guillain-Barre syndrome has been associated with Campylobacter jejuni and the generation of antibodies to microbial components that cross-react with epitopes on the surface of the neuron ${ }^{14}$.

Given the importance of the gut microbiome in immune function and autoimmune disease, for the present work we investigated the human gut microbiome in multiple sclerosis (MS). We identify alterations in the intestinal microbiota and find correlations with MS-associated immune changes and treatment. If further studies demonstrate that these candidate microorganisms play an active role in either contributing to or ameliorating MS, then there is the potential to develop new diagnostics and therapies to combat the disease.

\section{Results}

Subject characteristics. Faecal samples were collected from 60 MS patients and 43 healthy controls (Fig. 1); details of the study population are provided in Table 1 and in Methods. The MS and control cohorts had comparable demographic characteristics except that the MS cohort had an increased proportion of males. All MS patients had relapsing-remitting disease but none had an active relapse at the time of study enrollment.

Structure and composition of the gut microbiome in MS. Microbial DNA was extracted from faecal samples and 16S rRNA gene sequencing was performed on the Roche 454 and Illumina MiSeq platforms using primers targeting the V3-5 or the V4 variable regions, respectively. We used two sequencing platforms to avoid platform-specific biases and to provide complementary information: the Roche 454 platform produces longer sequencing reads but fewer reads, whereas the Illumina MiSeq provides shorter reads but greater sequencing depth. The resulting sequences were then processed using the mothur software package for quality filtering, removal of artifacts and clustering to operational taxonomic units (OTUs) ${ }^{15}$ (Supplementary Fig. 1). Roche 454 sequencing yielded $1,426,326$ reads of $\sim 450$ nucleotides each, with 426 OTUs identified after quality filtering. Illumina MiSeq sequencing yielded 11,498,168 pairedend reads of 150 nucleotides each, with 1,191 OTUs identified after filtering.

To assess overall differences in microbial community structure in MS patients and controls, we calculated measures of alpha- and beta-diversity. Alpha-diversity summarizes the microbial diversity within each sample, whereas beta diversity measures differences between samples. Shannon entropy, an alpha-diversity measurement of richness and evenness, was measured at multiple sequencing depths using rarefaction curves and was similar between MS patients and healthy controls (Supplementary Fig. 2). To determine whether overall microbial community structure was different between MS patients and controls, we calculated differences in beta-diversity using the weighted and unweighted UniFrac metric. Statistical analyses of the resulting matrices using the analysis of molecular variance technique did not reveal significant differences in overall microbial community structure between the two groups (Supplementary Fig. 3).

MS-associated microbiota changes at the phylum level. At the phylum level, the faecal microbiota of both groups was dominated by Firmicutes and Bacteroidetes, with smaller contributions of Euryarchaeota, Verrucomicrobia and Proteobacteria. The relative abundances of microbiota at the phylum level were compared between the entire MS cohort (both treated and untreated) and controls (Fig. 2). MS patients had a significantly increased relative abundance of the phyla Euryarchaeota and Verrucomicrobia compared to healthy controls (DESeq, Benjamini-Hochberg adjusted $P$ value $<0.05)$ by Roche 454 and Illumina sequencing (Fig. 2a, Table 2).

Because immunomodulatory therapy may skew microbiota composition, we separately analysed changes in the microbiota in untreated patients. Both Euryarchaeota and Verrucomicrobia were similarly elevated in untreated MS patients compared with controls, although changes in Euryarchaeota were only significant on the 454 platform, and trended in the same direction on the MiSeq platform. Consistent with other microbiota studies in humans, we detected inter-individual variability within control and MS patients. Thus, we provide rank abundance plots to depict phylum level abundances in each subject (Supplementary Fig. 4). 


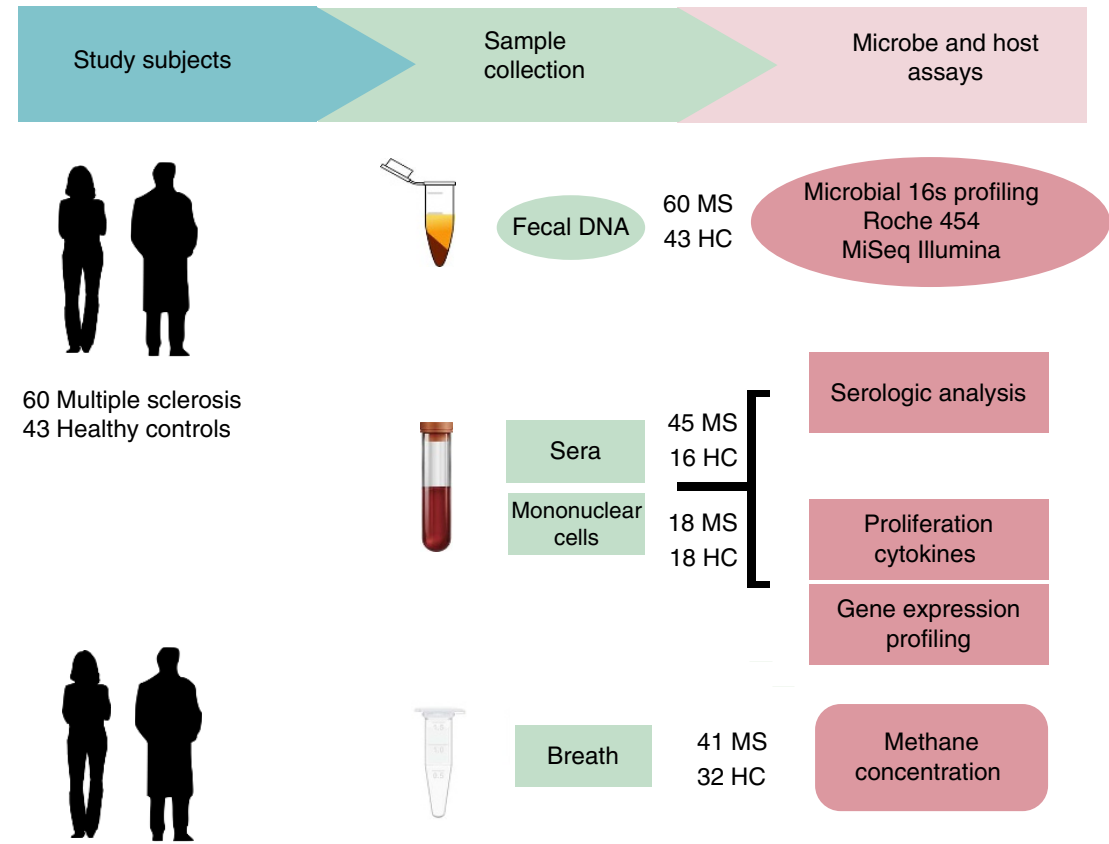

41 Multiple sclerosis 32 Healthy controls

Subject demographics

Dietary survey

Disease characteristics

Figure 1 | Study design. Faecal samples were collected from MS patients $(n=60)$ and healthy subjects $(n=43)$. Microbial DNA was extracted from frozen faecal samples and 16s rDNA sequencing was performed using Roche 454 and Illumina platforms. Gene expression profiling was performed on circulating monocytes and T cells from MS patients $(n=18)$ and healthy subjects $(n=18)$ using a Nanostring platform. Peripheral blood mononuclear cells were collected from MS patients $(n=18)$ and healthy subjects $(n=18)$ to conduct proliferation and cytokine assays in response to specific microbial stimulation. Sera from MS patients $(n=45)$ and healthy subjects $(n=16)$ was collected for ELISA-based techniques to capture serologic activity directed against specific microbes. Breath samples from MS patients $(n=41)$ and healthy subjects $(n=32)$ were collected from a second subject cohort to determine breath methane concentrations.

Table 1 | Demographics of study population.

\begin{tabular}{lcc} 
& Healthy & Multiple sclerosis \\
\cline { 2 - 3 } & $\mathbf{N}=\mathbf{4 3}$ & $\mathbf{N}=\mathbf{6 0}$ \\
\hline Age & $42.2 \pm 9.61$ & $49.7 \pm 8.50$ \\
Male (\%) & $6(14 \%)$ & $19(32 \%)$ \\
Female (\%) & $37(86 \%)$ & $41(68 \%)$ \\
Body mass index & $26.4 \pm 6.3$ & $27.2 \pm 4.7$ \\
Caucasian & 43 & 58 \\
Black & 0 & 2 \\
Hispanic & 0 & 1 \\
Disease Duration & NA & $12.8 \pm 8.3$ \\
EDSS Score & NA & $1.2 \pm 1.0$ \\
Untreated & NA & 28 \\
Beta-interferon & NA & 18 \\
Glatiramer acetate & NA & 14 \\
\hline NA, not applicable & \multicolumn{2}{|}{} \\
\hline
\end{tabular}

MS-associated microbiota changes at the genus level. We next investigated whether relative abundances of the microbiota differed between MS (untreated and treated) patients and controls at the genus level (Fig. 2b,c). The relative abundances of Methanobrevibacter, a genus in the phylum Euryarchaeota, and Akkermansia, a genus in the phylum Verrucomicrobia, were both increased in MS patients compared with controls by Roche 454 sequencing and by Illumina sequencing (Fig. 2c, Table 2). Furthermore, Butyricimonas, which belongs to the phylum Bacteroidetes had a reduced relative abundance in MS as detected by both sequencing platforms. These changes were similarly detected in untreated MS patients compared with controls (Fig. 2c, Supplementary Fig. 5). In addition Collinsella and Slackia, both belonging to the phylum Actinobacteria, and Prevotella, belonging to the phylum Bacteroidetes was decreased in untreated MS patients as detected by both sequencing platforms (Table 2; Supplementary Fig. 6). In general, the results from the two sequencing platforms were concordant, with $98 \%$ of the total microbial abundance composed of shared genera. While we were able to detect some primer bias (for example, higher detection of Akkermansia with the MiSeq V4 strategy compared with the 454 V35 strategy) the MS-related changes generally remained the same (Supplementary Fig. 7).

Effect of therapy on gut microbiota. We then asked whether immunomodulatory therapy was associated with an altered microbiota in treated versus untreated MS patients. We found that treated patients had increases in the Prevotella and Sutterella as detected by Roche 454 and by Illumina sequencing (Fig. 2c, Table 2, Supplementary Fig. 8). Since these genera are either significantly reduced or show a trend of reduced 
a
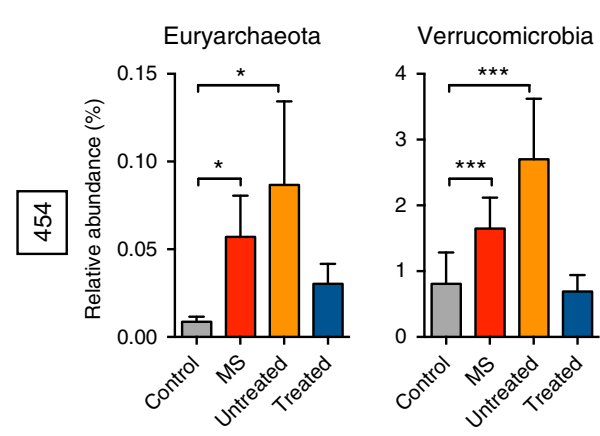

Euryarchaeota

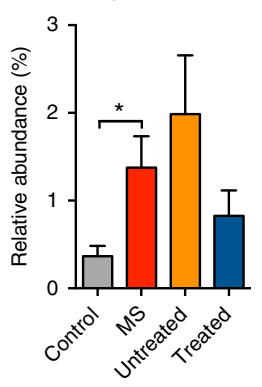

Verrucomicrobia

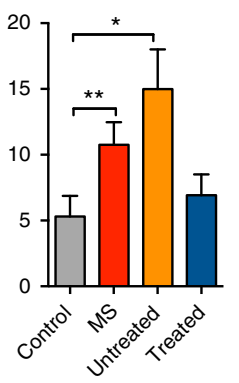

b

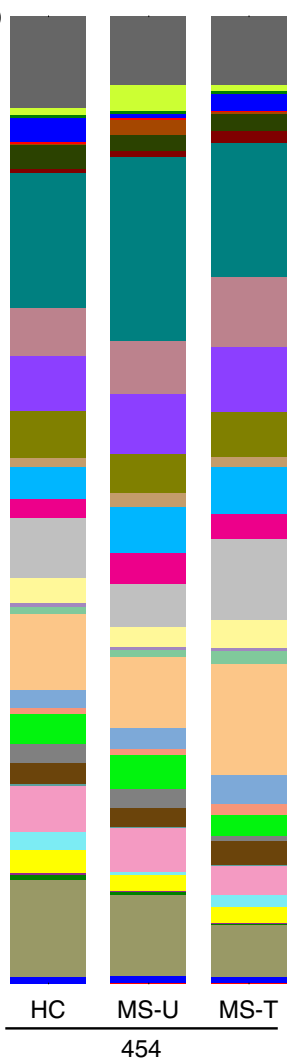

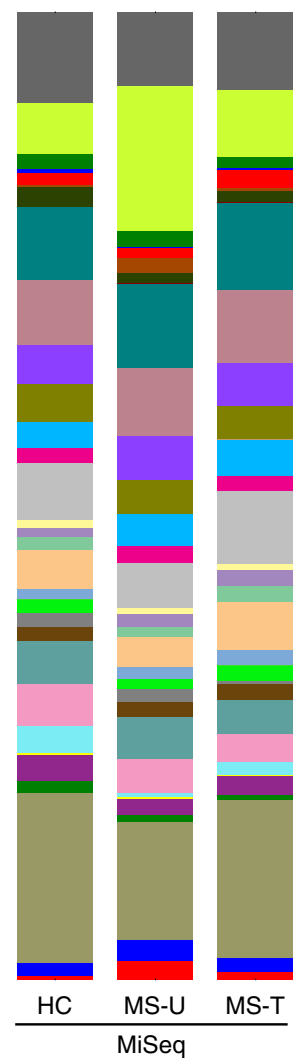

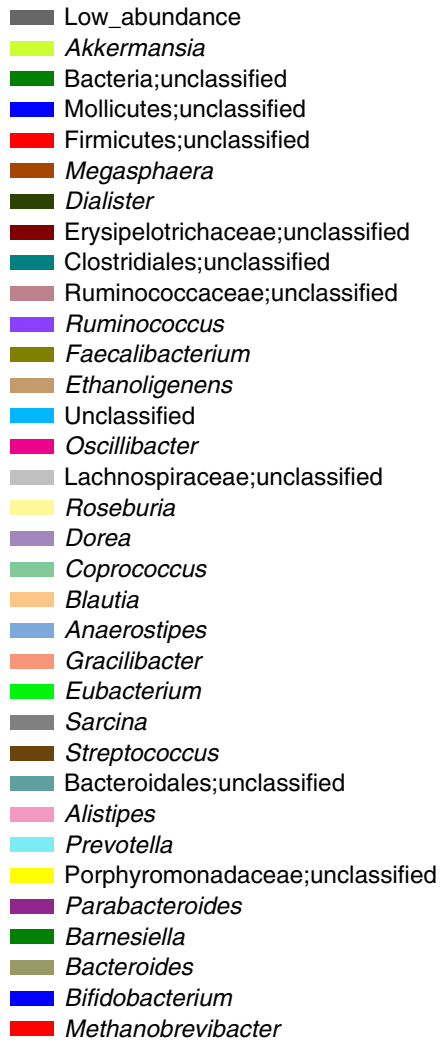

C
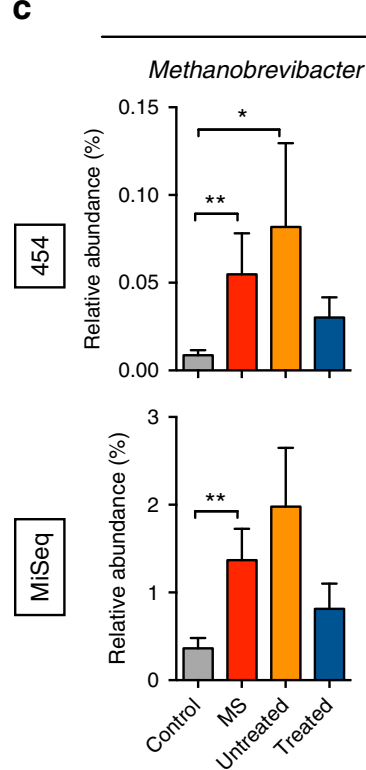

MS-effect
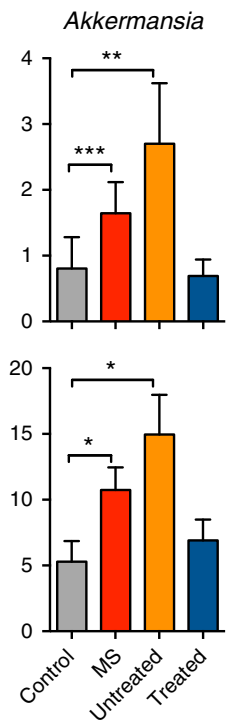

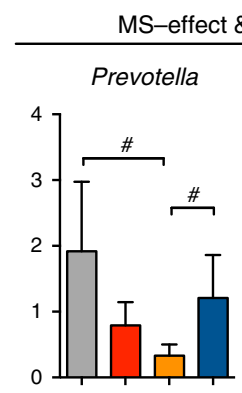

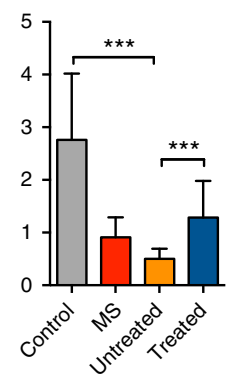
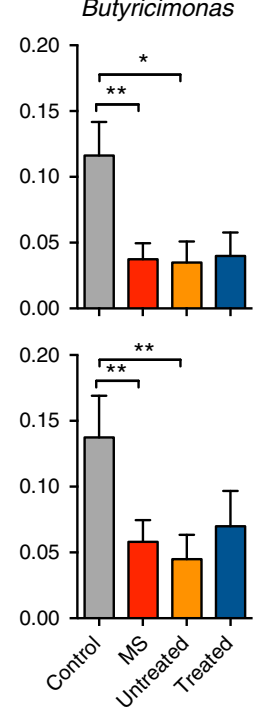

Treatment-effect
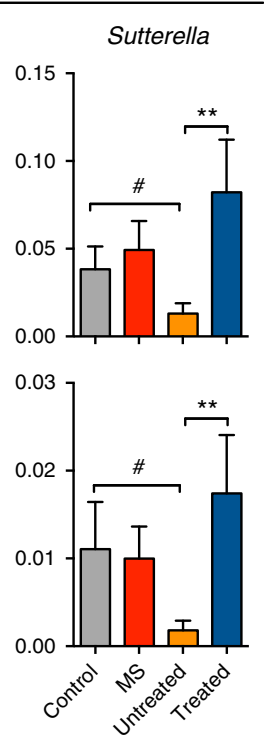

Treatment-effect

Sarcina
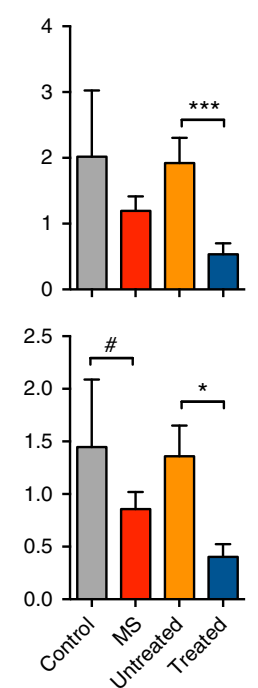

Figure 2 | Compositional differences in faecal microbiota between MS patients and healthy subjects. (a) Relative abundances of Euryarchaeota and Verrucomicrobia in the faecal microbiota of healthy controls $(n=43$, grey bar), all MS patients $(n=60$, red) and both untreated ( $n=28$, orange) and treated MS patient ( $n=32$, blue) subgroups as analysed by two independent sequencing technologies, 454 (top) or MiSeq (bottom). (b) Relative abundance of prevalent microbiota ( $>1 \%$ in any sample group) determined from MiSeq and 454 high-throughput sequencing. (c) Relative abundances of genera in the faecal microbiota that are significantly altered between healthy controls $(n=43)$ and MS patients $(n=60 ;$ MS-effect) or between untreated $(n=28)$ and treated MS patients $(n=32)$ (disease effect) as analysed by two independent sequencing technologies. Significance was determined by DESeq and Benjamini-Hochberg corrected $P$ values $<0.05$ with a false discovery rate threshold of 0.1. Bars represent average, and error bars depict s.e.

populations in untreated patients compared with controls, it suggests that treatment with immunomodulatory therapy may normalize some of the MS-related changes in the microbiota. The genus Sarcina was reduced in treated versus untreated MS patients by both sequencing platforms (Fig. 2b,c).
However, Sarcina levels were similar between untreated patients and controls, suggesting a treatment-associated effect. No significant differences in the microbiota were noted when MS patients treated with interferon therapy were compared with those treated with glatiramer acetate, although the sample sizes of 
Table 2 | Multiple sclerosis and MS-treatment-associated taxa.

\begin{tabular}{|c|c|c|c|c|c|c|c|c|c|c|c|c|c|c|}
\hline & \multicolumn{8}{|c|}{ Relative abundances } & \multicolumn{6}{|c|}{ BH corrected $P$ values } \\
\hline & \multicolumn{4}{|c|}{454} & \multicolumn{4}{|c|}{ Illumina } & \multicolumn{2}{|c|}{ HC vs MS } & \multicolumn{2}{|c|}{ HC versus Un } & \multicolumn{2}{|c|}{ Tr vs Untreated } \\
\hline & HC & All MS & Untr MS & Tr MS & HC & All MS & Untr MS & Tr MS & 454 & MiSeq & 454 & MiSeq & 454 & MiSeq \\
\hline \multicolumn{15}{|l|}{ Phyla } \\
\hline Euryarcheota & $8.65 \times 10^{-5}$ & $5.48 \times 10^{-4}$ & $3.36 \times 10^{-4}$ & $3.12 \times 10^{-4}$ & $3.66 \times 10^{-3}$ & $1.38 \times 10^{-2}$ & $1.17 \times 10^{-2}$ & $8.53 \times 10^{-3}$ & $1.38 \times 10^{-2}$ & $2.74 \times 10^{-2}$ & $1.88 \times 10^{-2}$ & n.s. & n.s. & n.s. \\
\hline Verrucomicrobia & $8.06 \times 10^{-3}$ & $1.65 \times 10^{-2}$ & $2.25 \times 10^{-2}$ & $5.80 \times 10^{-3}$ & $5.30 \times 10^{-2}$ & $1.07 \times 10^{-1}$ & $1.39 \times 10^{-1}$ & $6.18 \times 10^{-2}$ & $1.77 \times 10^{-5}$ & $3.66 \times 10^{-3}$ & $8.91 \times 10^{-4}$ & $1.41 \times 10^{-2}$ & n.s. & n.s. \\
\hline \multicolumn{15}{|l|}{ Genera } \\
\hline Methanobrevibacter & $8.65 \times 10^{-5}$ & $5.48 \times 10^{-4}$ & $3.37 \times 10^{-4}$ & $3.12 \times 10^{-4}$ & $3.64 \times 10^{-3}$ & $1.37 \times 10^{-2}$ & $1.16 \times 10^{-2}$ & $8.40 \times 10^{-3}$ & $7.80 \times 10^{-3}$ & $2.30 \times 10^{-3}$ & $1.28 \times 10^{-2}$ & n.s. & n.s. & n.s. \\
\hline Akkermansia & $8.06 \times 10^{-3}$ & $1.65 \times 10^{-2}$ & $2.25 \times 10^{-2}$ & $5.80 \times 10^{-3}$ & $5.29 \times 10^{-2}$ & $1.07 \times 10^{-1}$ & $1.39 \times 10^{-1}$ & $6.18 \times 10^{-2}$ & $9.00 \times 10^{-4}$ & $3.45 \times 10^{-2}$ & $3.20 \times 10^{-3}$ & $3.36 \times 10^{-2}$ & n.s. & n.s. \\
\hline Butyricimonas & $1.16 \times 10^{-3}$ & $3.75 \times 10^{-4}$ & $2.80 \times 10^{-4}$ & $4.11 \times 10^{-4}$ & $1.37 \times 10^{-3}$ & $5.81 \times 10^{-4}$ & $4.26 \times 10^{-4}$ & $7.21 \times 10^{-4}$ & $3.50 \times 10^{-3}$ & $9.50 \times 10^{-3}$ & $1.31 \times 10^{-2}$ & $6.70 \times 10^{-3}$ & n.s. & n.s. \\
\hline Paraprevotella & $3.57 \times 10^{-4}$ & $2.18 \times 10^{-5}$ & $\begin{array}{c}2.80 \times 10 \\
0\end{array}$ & $3.84 \times 10^{-5}$ & $3.47 \times 10^{-3}$ & $4.65 \times 10^{-4}$ & $3.44 \times 10^{-5}$ & $7.73 \times 10^{-4}$ & $3.45 \times 10^{-2}$ & $8.70 \times 10^{-3}$ & $9.52 \times 10^{-2}$ & $1.50 \times 10^{-3}$ & $\begin{array}{l}\text { n.ts. } \\
\text { n.s. }\end{array}$ & $\begin{array}{l}\text { n.s. } \\
\text { n.s. }\end{array}$ \\
\hline Haemophilus & $3.95 \times 10^{-3}$ & $3.32 \times 10^{-3}$ & $5.25 \times 10^{-3}$ & $2.68 \times 10^{-3}$ & $4.61 \times 10^{-3}$ & $4.79 \times 10^{-3}$ & $7.75 \times 10^{-3}$ & $3.83 \times 10^{-3}$ & $3.50 \times 10^{-3}$ & $2.57 \times 10^{-2}$ & $2.10 \times 10^{-3}$ & $9.60 \times 10^{-3}$ & & $6.00 \times 10^{-}$ \\
\hline Slackia & $1.99 \times 10^{-3}$ & $5.50 \times 10^{-4}$ & $5.00 \times 10^{-4}$ & $5.56 \times 10^{-4}$ & $1.62 \times 10^{-4}$ & $4.77 \times 10^{-5}$ & $6.17 \times 10^{-5}$ & $4.40 \times 10^{-5}$ & $3.60 \times 10^{-3}$ & $5.21 \times 10^{-2}$ & $2.5 \times 10^{-5}$ & $1.35 \times 10^{-2}$ & $6.55 \times 10^{-2}$ & n.s. \\
\hline Collinsella & $5.54 \times 10^{-3}$ & $4.90 \times 10^{-3}$ & $3.61 \times 10^{3}$ & $6.10 \times 10^{3}$ & $2.27 \times 10^{3}$ & $1.52 \times 10^{-3}$ & $1.16 \times 10^{-3}$ & $1.89 \times 10^{-3}$ & $\begin{array}{l}\text { n.s. } \\
\text { n. }\end{array}$ & n.s. & $8.19 \times 10^{-2}$ & $4.30 \times 10^{-3}$ & $\begin{array}{c}0.35 \times 10 \\
\text { n.s. }\end{array}$ & $9.20 \times 10^{-1}$ \\
\hline Megasphaera & $1.29 \times 10^{-3}$ & $8.18 \times 10^{-3}$ & $2.05 \times 10^{-2}$ & $1.57 \times 10^{-3}$ & $1.46 \times 10^{-3}$ & $8.67 \times 10^{-3}$ & $2.06 \times 10^{-2}$ & $2.45 \times 10^{-3}$ & $9.50 \times 10^{-3}$ & n.s. & $1.00 \times 10^{-4}$ & $3.84 \times 10^{-2}$ & $1.90 \times 10^{-7}$ & $5.50 \times 10^{-}$ \\
\hline Cloacibacillus & $4.26 \times 10^{-5}$ & $2.64 \times 10^{-4}$ & $5.93 \times 10^{-4}$ & $4.41 \times 10^{-5}$ & 0 & $1.06 \times 10^{-5}$ & $2.34 \times 10^{-5}$ & $1.50 \times 10^{-6}$ & $3.20 \times 10^{-2}$ & n.s. & $4.98 \times 10^{-2}$ & $9.20 \times 10^{-3}$ & $6.92 \times 10^{-2}$ & $3.45 \times 10$ \\
\hline Veillonellaceae_unc & $8 \times 10^{-3}$ & $6.12 \times 10^{-4}$ & $9.21 \times 10^{-4}$ & $3.36 \times 10^{-4}$ & $1.14 \times 10^{-3}$ & $1.91 \times 10^{-4}$ & $3.56 \times 10^{-4}$ & $1.12 \times 10^{-4}$ & $\begin{array}{l}\text { n.s. } \\
\text { n.s. }\end{array}$ & n.s. & $8.51 \times 10^{-2}$ & $2.38 \times 10^{-2}$ & $5.17 \times 10^{-2}$ & $3.45 \times 10$ \\
\hline Prevotella & $1.92 \times 10^{-2}$ & $7.90 \times 10^{-3}$ & $1.23 \times 10^{-3}$ & $1.25 \times 10^{-2}$ & $2.76 \times 10^{-2}$ & $3.06 \times 10^{-3}$ & $1.23 \times 10^{-3}$ & $1.32 \times 10^{-2}$ & n.s. & n.s. & $7.27 \times 10^{-2}$ & $2.80 \times 10^{-7}$ & $9.02 \times 10^{-2}$ & $2.80 \times 10$ \\
\hline Sutterella & $6.40 \times 10^{-3}$ & $6.49 \times 10^{-3}$ & $1.16 \times 10^{-4}$ & $8.49 \times 10^{-4}$ & $1.11 \times 10^{-4}$ & $1.00 \times 10^{-4}$ & $1.54 \times 10^{-5}$ & $1.80 \times 10^{-4}$ & n.s. & n.s. & $7.64 \times 10^{-2}$ & $6.80 \times 10^{-2}$ & $4.20 \times 10^{-3}$ & $8.70 \times 10^{-}$ \\
\hline Clostridia_unc & $5.05 \times 10^{-5}$ & $1.77 \times 10^{-4}$ & $3.17 \times 10^{-4}$ & $5.25 \times 10^{-5}$ & $51 \times 10^{-2}$ & $8.51 \times 10^{-2}$ & $8.51 \times 10^{-2}$ & $9.07 \times 10^{-2}$ & $8.51 \times 10^{-2}$ & & $8.00 \times 10^{-3}$ & n.s. & $1.92 \times 10^{-2}$ & $8.60 \times 10^{-}$ \\
\hline Sarcina_-_urte & $2.02 \times 10^{-2}$ & $1.20 \times 10^{-2}$ & $2.04 \times 10^{-2}$ & $5.45 \times 10^{-3}$ & $1.45 \times 10^{-2}$ & $8.58 \times 10^{-3}$ & $1.51 \times 10^{-2}$ & $4.11 \times 10^{-3}$ & $6.92 \times 10^{-2}$ & $\begin{array}{l}\text { n.s. } \\
\text { n.s. }\end{array}$ & $\begin{array}{l}0.00 \times 10 \\
\text { n.s. }\end{array}$ & $\begin{array}{l}\text { n.t. } \\
\text { n.s. }\end{array}$ & $9.00 \times 10^{-4}$ & $2.52 \times 10^{-2}$ \\
\hline Mollicutes_unc & $2.49 \times 10^{-2}$ & $1.05 \times 10^{-2}$ & $2.02 \times 10^{-3}$ & $2.02 \times 10^{-3}$ & $3.61 \times 10^{-3}$ & $1.08 \times 10^{-3}$ & $7.81 \times 10^{-4}$ & $1.36 \times 10^{-3}$ & $8.30 \times 10^{-3}$ & n.s. & $3.00 \times 10^{-7}$ & n.s. & n.s. & n.s. \\
\hline Prevotellaceae_unc & $4.99 \times 10^{-3}$ & $1.37 \times 10^{-4}$ & $7.31 \times 10^{-6}$ & $1.07 \times 10^{-5}$ & $2.15 \times 10^{-3}$ & $4.22 \times 10^{-4}$ & $1.40 \times 10^{-4}$ & $2.37 \times 10^{-4}$ & $3.00 \times 10^{-10}$ & $\begin{array}{l}\text { n.s. } \\
\text { n.s. }\end{array}$ & $4.40 \times 10^{-5}$ & n.s. & n.s. & n.s. \\
\hline Holdemania & $1.72 \times 10^{-3}$ & $3.80 \times 10^{-3}$ & $3.69 \times 10^{-3}$ & $3.08 \times 10^{-3}$ & $4.26 \times 10^{-4}$ & $4.85 \times 10^{-4}$ & $4.03 \times 10^{-4}$ & $5.51 \times 10^{-4}$ & $4.20 \times 10^{-3}$ & n.s. & $4.02 \times 10^{-2}$ & n.s. & n. & n.s. \\
\hline Desulfovibrio & $9.48 \times 10^{-5}$ & $1.63 \times 10^{-4}$ & $3.75 \times 10^{-4}$ & $3.95 \times 10^{-5}$ & $1.16 \times 10^{-3}$ & $9.31 \times 10^{-4}$ & $1.65 \times 10^{-3}$ & $4.65 \times 10^{-4}$ & $\begin{array}{l}\text { n.s. } \\
\text { n.s. }\end{array}$ & $\begin{array}{l}\text { n.s. } \\
\text { n.s. }\end{array}$ & $1.58 \times 10^{-2}$ & $\begin{array}{l}\text { n.s. } \\
\text { n.s. }\end{array}$ & $1.80 \times 10^{-3}$ & $\begin{array}{l}\text { n.t. } \\
\text { n.s. }\end{array}$ \\
\hline Peptococcaceae unc & $8.14 \times 10^{-5}$ & $1.74 \times 10^{-4}$ & $3.85 \times 10^{-4}$ & $5.26 \times 10^{-5}$ & $2.01 \times 10^{-4}$ & $2.72 \times 10^{-4}$ & $2.56 \times 10^{-4}$ & $2.79 \times 10^{-4}$ & $\begin{array}{l}\text { n.t.s. } \\
\text { n.s. }\end{array}$ & n.s & $4.03 \times 10^{-2}$ & n.s. & $3.30 \times 10^{-2}$ & n.s. \\
\hline Barnesie & $4.76 \times 10^{-3}$ & $2.00 \times 10^{-3}$ & $1.35 \times 10^{-3}$ & $1.41 \times 10^{-3}$ & $1.18 \times 10^{-2}$ & $6.55 \times 10^{-3}$ & $8.35 \times 10^{-3}$ & $5.43 \times 10$ & n. & $1.00 \times 10^{-4}$ & $1.60 \times 10^{-3}$ & n.s. & n.s. & n.s. \\
\hline $\begin{array}{l}\text { Barressella } \\
\text { Acidaminococcaceae unc }\end{array}$ & $\begin{array}{l}4.16 \times 10^{-3} \\
3.45 \times 10^{-3}\end{array}$ & $8.43 \times 10^{-4}$ & $1.36 \times 10^{-4}$ & $5.01 \times 10^{-4}$ & $5.70 \times 10^{-4}$ & $5.82 \times 10^{-5}$ & $4.14 \times 10^{-5}$ & $8.51 \times 10^{-5}$ & $\begin{array}{l}\text { n.ts. } \\
\text { n.s. }\end{array}$ & $2.57 \times 10^{-2}$ & $3.15 \times 10^{-2}$ & n.s. & n.s. & n.s. \\
\hline Megamonas & $8.54 \times 10^{-5}$ & $6.34 \times 10^{-5}$ & $\begin{array}{c}1.40 \times 10 \\
0\end{array}$ & $1.25 \times 10^{-4}$ & $1.20 \times 10^{-4}$ & $2.79 \times 10^{-4}$ & $2.66 \times 10^{-6}$ & $5.47 \times 10^{-4}$ & $\begin{array}{l}\text { n.t. } \\
\text { n.s. }\end{array}$ & $1.80 \times 10^{-5}$ & $\begin{array}{l}\text { n.s. } \\
\text { n.t. }\end{array}$ & $\begin{array}{c}\text { n.s. } \\
1.54 \times 10^{-2}\end{array}$ & $\begin{array}{l}\text { n.t. } \\
\text { n.s. }\end{array}$ & n.s. \\
\hline Guggenheimella & - & $0.34 \times$ & - & $1.25 \times$ & $3.17 \times 10^{-3}$ & $1.62 \times 10^{-3}$ & $1.94 \times 10^{-5}$ & $3.16 \times 10^{-3}$ & - & $3.60 \times 10^{-5}$ & - & $9.50 \times 10^{-3}$ & - & n.s. \\
\hline Thermoplasmatales_unc & - & - & - & - & $1.82 \times 10^{-6}$ & $2.08 \times 10^{-5}$ & $2.63 \times 10^{-5}$ & $1.45 \times 10^{-6}$ & - & $3.45 \times 10^{-2}$ & - & $1.23 \times 10^{-2}$ & - & $1.62 \times 10$ \\
\hline Buttiauxella & $7.77 \times 10^{-5}$ & $2.45 \times 10^{-4}$ & $2.01 \times 10^{-4}$ & $2.77 \times 10^{-4}$ & $3.32 \times 10^{-3}$ & $1.32 \times 10^{-3}$ & $1.41 \times 10^{-4}$ & $2.49 \times 10^{-3}$ & n.s. & n.s. & n.s. & $1.00 \times 10^{-3}$ & n.s. & $1.90 \times 10^{-}$ \\
\hline Sporanaerobacter & - & $2.45 \times 10$ & $2.01 \times 10$ & - & $8.78 \times 10^{-4}$ & $6.45 \times 10^{-4}$ & $1.43 \times 10^{-4}$ & $1.04 \times 10^{-3}$ & - & n.s. & - & $4.20 \times 10^{-4}$ & - & $1.00 \times 10$ \\
\hline
\end{tabular}

HC, healthy controls; MS, multiple sclerosis; unc, unclassified; Untr, untreated MS patients; Tr, treated MS patients.

The table lists taxa that differ between $\mathrm{HC}$ and MS patients, between $\mathrm{HC}$ and Untr and between untreated and Tr, detected on two different sequencing platforms, 454 and MiSeq. Significant differences were tested by DESeq, and $P$ values were adjusted by the Benjamini-Hochberg method. Relative abundances are listed. Values that are significant $(P<0.05)$ on both platforms are in bold. Taxa are listed at

the genus level or at their lowest possible level of classification, followed by unc. Genera that were not detected on a given platform are indicated with a ( - ). Non-significant $P$ values are listed as n.s.

these subsets were not sufficiently powered to evaluate this comparison.

To determine whether the differences we observed were related to age, gender or body mass index (BMI), we reanalysed our data using a multi-factorial model. For all MS patients (treated and untreated) compared with controls, the differences in relative abundances for the genera Methanobrevibacter, Akkermansia and Butyricimonas remained significant with at least one of the sequencing platforms. We also found that treatment-related differences in relative abundances for the genera Prevotella, Sutterella and Sarcina remained significant.

Phylogenetic placement of $16 \mathrm{~S}$ rRNA sequences. Validating bacterial identification is important to select the optimal bacteria to monitor in translational studies, however, partial 16S rRNA reads do not always provide sufficient information for identification to the species level. Thus, phylogenetic placement of $16 \mathrm{~S}$ rRNA sequences was used to further identify taxa of interest and to assess the accuracy of identification using pplacer ${ }^{16}$ (Supplementary Figs 9 and 10). The representative sequence from the most prevalent OTU was used for each taxa of interest. The Methanobrevibacter sequence from our study placed most closely to reference $M$. smithii (placement likelihood $(\mathrm{PL})=0.94$ ), Akkermansia placed most closely to A. muciniphila $(\mathrm{PL}=1.00)$, and Butyricimonas had a greater likelihood of identification as $B$. synergistica $(\mathrm{PL}=0.83)$ than $B$. virosa $(\mathrm{PL}=0.50)$. For genera found to be different between untreated MS patients and controls, Collinsella placed most closely to $C$. aerofaciens $(\mathrm{PL}=0.85)$, Slackia placed closer to $S$. isoflavoniconvertens $(\mathrm{PL}=1.0$ than to $S$. piriformis $(\mathrm{PL}=0.76)$, Prevotella placed closer to $P$. stercorea $(\mathrm{PL}=1.0)$ than to two different $P$. copri reference strains $(P L=0.76$ and 0.81$)$. For taxa found to be different between untreated and treated MS patients, Sutterella could not be resolved between $S$. stercoricanis or $S$. wadsworthensis $(\mathrm{PL}=1.0$ and 1.0, respectively) and sequences mapped to the genus Sarcina placed closest to $S$. ventriculi (two sequences placing near $S$. ventriculi with likelihoods of 0.66 and 0.85 ). In total, pplacer was able to identify the most likely genus and species from currently available reference databases.
Gene expression in peripheral blood $\mathrm{T}$ cells and monocytes. We used the Nanostring immunology panel to measure the expression of 568 immune-related genes from peripheral blood derived $\mathrm{CD}^{+}{ }^{+} \mathrm{T}$ cells and $\mathrm{CD} 14^{+}$monocytes from a subset of 18 MS patients and 18 controls subjects. Because Methanobrevibacter was significantly increased in MS patients compared with healthy subjects, and since Methanobrevibacter has been previously implicated as a proinflammatory microbe ${ }^{17}$, we selected MS patients and healthy subjects from our cohort that had the highest and lowest populations of Methanobrevibacter. The demographics and disease characteristics of MS patients and healthy subjects within this subset were similar to those of the larger group (Supplementary Table 1).

We found unique immune transcriptional profiles in $\mathrm{T}$ cells and monocytes from MS patients compared with healthy controls (Supplementary Fig. 11a-c). Ingenuity pathway analysis was used to identify altered canonical pathways. Both $\mathrm{T}$ cells and monocytes were predicted to have activated interferon, NF-KB, Toll-like receptor and IL-6 signalling pathways, and decreased PPAR $\alpha / R X R \alpha$, consistent with pathways previously correlated with $\mathrm{MS}^{18-23}$ (Fig. 3a, Supplementary Fig. 11d).

We then investigated potential associations between the MS-related microbiota and immune changes. We correlated microbial abundance with a set of significantly altered genes biologically curated from the identified canonical pathways (Fig. 3b). For $\mathrm{T}$ cells from all subjects, we found that both Methanobrevibacter and Akkermansia had positive correlations with CASP1, TRAF5 and STAT5B, while Butyricimonas had negative correlations with these genes (Fig. 3b), which are implicated in IFN signalling, IL-2 signalling, and PPAR and RXR activation. Because the correlations between microbial populations and immune expression levels could driven by the disease, we examined the correlations within untreated MS patients and within healthy controls separately. Among untreated MS patients, we again noted that Methanobrevibacter and Akkermansia had positive correlations and Butyricimonas had negative correlations with this set of genes, while the correlations were near zero among healthy controls alone. Methanobrevibacter and Akkermansia also had negative correlations with TNFA1P3, 
a known potent anti-inflammatory cytokine in autoimmune demyelination, along with NFKBIA previously known to be underexpressed in $\mathrm{MS}^{20,21}$. Butyricimonas had positive correlations with these two genes (Fig. 3b).

In monocytes from MS patients, Methanobrevibacter and Akkermansia had positive correlations with MAPK14, MAPK1, LTBR, STAT5B, CASP1 and HLA-DRB1, while Butyricimonas had negative correlations with these genes, which are implicated in dendritic cell maturation, IFN signalling and TREM signalling pathways. Among untreated MS patients, Akkermansia and Butyricimonas had positive and negative correlations, respectively, with this set of genes, whereas these effects were not observed in controls (Fig. 3b). Methanobrevibacter and Akkermansia also had negative correlations with HLA-A, HLA-B and BCL2 in untreated MS patients.

Since we observed correlations between the abundance of specific microbes and gene expression in monocytes and $\mathrm{T}$ cells from MS patients, we were interested in determining whether these organisms might drive an altered immunologic response in MS patients and controls. Because lipids derived from Methanobrevibacter are reported to have adjuvant effects ${ }^{24}$, we investigated Methanobrevibacter smithii induced proliferation and cytokine production in human peripheral blood mononuclear cells (PBMCs), but found no difference in the response to Methanobrevibacter in MS patients versus controls or in subjects with high Methanobrevibacter in the gut (Supplementary Fig. 12).

Relationship of the gut microbiome to humoral responses. We then examined whether sera from MS patients and healthy subjects contained antibodies reactive to Methanobrevibacter or components of Methanobrevibacter. We found antiMethanobrevibacter IgM, IgG and IgA antibodies (titre $>1: 64$ ) as measured by enzyme-linked immunosorbent assay (ELISA) against lysates of Methanobrevibacter in 33\% of MS patients and $28 \%$ of controls, with no differences in anti-Methanobrevibacter antibody titres between the two groups.

Breath methane in MS. Because Methanobrevibacter is the dominant methane-producing microbe that inhabits the human gastrointestinal tract, the likely presence of Methanobrevibacter in the gut can be measured indirectly by a breath test, which assesses exhaled methane by gas chromatography ${ }^{25}$. Detection of $>1$ parts-per-million of methane in the breath reflects the presence of at least $10^{7}-10^{8}$ methanogens per gram of stool and has been shown to be driven by Methanobrevibacter smithii ${ }^{26}$. After we discovered increased Methanobrevibacter in the gut by 16S rRNA sequencing, we performed a methane breath test on a second cohort of $41 \mathrm{MS}$ patients and 32 controls to determine whether we could verify the presence of methane-producing organisms by a simple and independent in vivo measurement. The second cohort was chosen to resemble our first cohort (Supplementary Table 2). We detected the presence of methane in $13 / 41$ MS patients versus. $8 / 32$ controls. MS patients had elevated levels of breath methane versus controls $(8.07 \pm 2.46$ versus $1.65 \pm 0.93 \mathrm{ppm},\left(t\right.$-test, $P$ value $\left.<1.81 \times 10^{-2}\right)$, consistent with our observation of increased methanogens in the gut of MS patients (Fig. 4).

\section{Discussion}

Host-commensal interactions have increasingly been shown to play a role in the induction of autoimmunity both in experimental animals and human diseases including inflammatory bowel disease, rheumatoid arthritis, type 1 diabetes and experimental autoimmune encephalomyelitis $8,27,28$. The origin of a

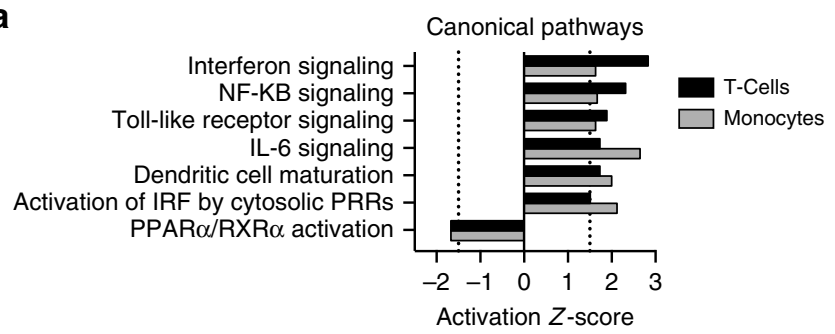

b
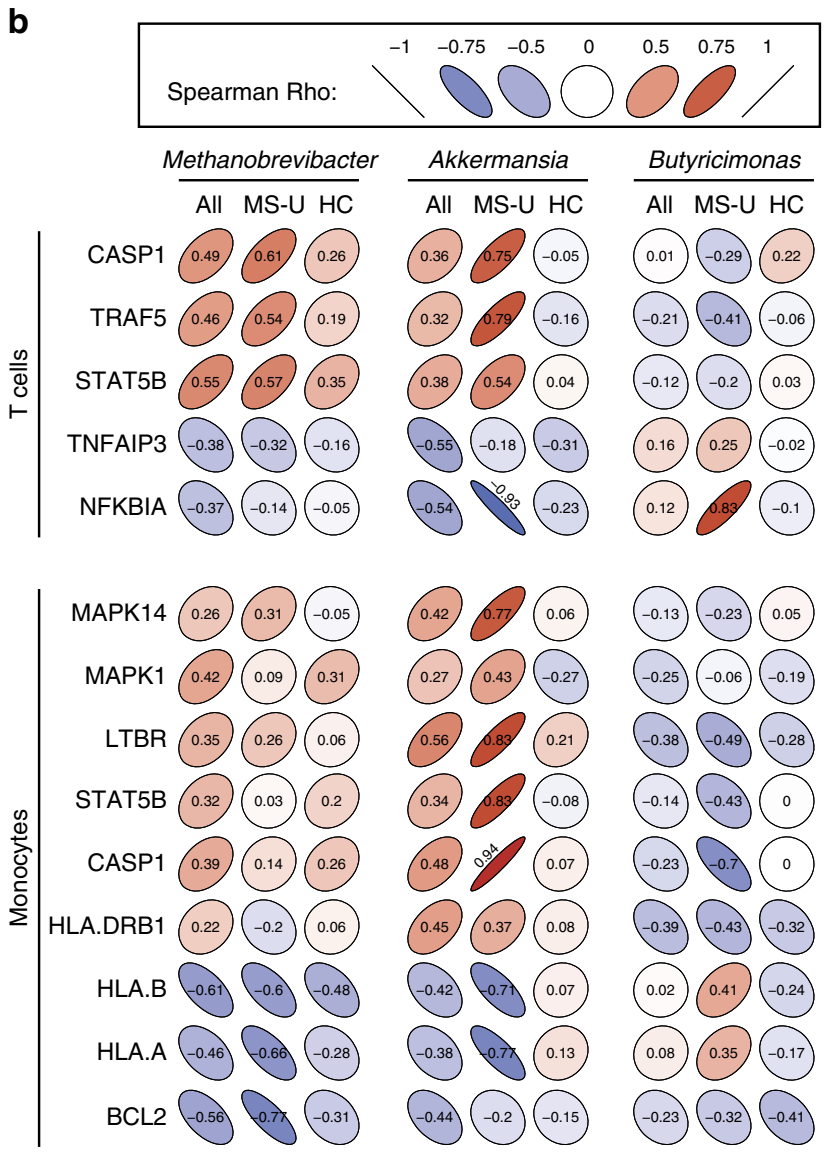

Figure 3 | Correlations between microbiota abundances and immune gene expression. (a) Gene expression was measured from circulating T cells and monocytes by the Nanostring Immunology panel in MS patients $(n=18)$ and healthy controls $(n=18)$. (a) canonical pathways significantly altered in MS patients and healthy subjects with an activation $z$-score $>|1.5|$ in both T cells (black bars) and monocytes (grey bars) identified by Ingenuity Pathway Analysis. (b) Altered gut microbiota abundances correlate with immune gene expression in MS patients. Spearman's correlations $(\sigma)$ between the relative abundance of significantly altered microbes in subject groups and the relative expression of genes from identified canonical pathways significantly altered between healthy controls and untreated MS patients. Colour and slope of ellipse indicate magnitude of correlation, with $\sigma$ value superimposed on ellipse. Subject groups were either all MS patients and controls together (All), untreated MS patients alone (MS-U) or healthy controls alone (HC).

the autoimmune process in multiple sclerosis is still poorly understood and whether the inciting factors that trigger inflammation primarily occur in the central nervous system or in the periphery is unknown. Given that disease concordance in MS is $25 \%$ in monozygotic twins, both genetic and environmental factors likely contribute to the development of disease ${ }^{29}$, and the gut microbiota might be one such environmental factor. 
We undertook studies to define the community structure of the faecal microbiome in MS patients using high-throughput $16 \mathrm{~S}$ rRNA gene sequencing. We found alterations at the phylum level with increases in Euryarchaeota and Verrucomicrobia. At the genus level, more specific shifts were observed, including increases of Methanobrevibacter and Akkermansia and reduction in Butyricimonas. Given that discrepancies may occur due to variability in primer selection and sequencing reads, we utilized two separate sequencing methodologies and observed that the majority of taxonomic shifts were consistent in both platforms.

Several of the organisms identified in this study as being altered in MS have been demonstrated to drive inflammation or have been associated with autoimmunity. The archaeon Methanobrevibacter has been implicated in inflammation by its capacity to recruit inflammatory cells and activate human dendritic cells ${ }^{17}$ and its role in inflammatory diseases, including periodontitis, asthma and inflammatory bowel disease ${ }^{24,30,3}$. In addition, archaeosomes derived from Methanobrevibacter have potent adjuvant properties secondary to their unique lipid structure and have been used as adjuvants for vaccines ${ }^{32}$. Methanobrevibacter is distributed throughout the small bowel and colon and is tightly adherent to the mucosa via regulated expression of adhesin-like proteins, placing it in close proximity to the gut-associated lymphoid tissue ${ }^{33}$. Consequently, when mucosa-associated (rather than luminal) microbiota were studied in inflammatory bowel disease, Methanobrevibacter smithii was increased more than threefold in mucosal samples from patients with both Crohn's disease and ulcerative colitis compared to controls $^{30}$. Methanobrevibacter smithii is also recovered more frequently in children with obesity, a known risk factor for the development of MS in adult life ${ }^{34}$. Furthermore, in a pilot study of pediatric multiple sclerosis, children colonized with Methanobrevibacter had a shorter time to relapse ${ }^{35}$.

We also found that the phylum Verrucomicrobia was increased in MS patients and was driven by the genus Akkermansia, which was also reported in a pilot study of 7 MS patients ${ }^{36}$. In contrast to our findings in MS, Akkermansia species have been reported to be decreased in other autoimmune diseases including psoriatic arthritis ${ }^{37}$. Akkermansia has been reported to have both regulatory and inflammatory properties, and is a mucin-degrader that converts mucin to short-chain fatty acids that may mediate the immunoregulatory effects ${ }^{38}$. Alternatively, Akkermansia has been correlated to proinflammatory pathways including upregulation of genes involved in antigen-presentation, B- and T-cell receptor signalling, and activation of complement and coagulation cascades ${ }^{39}$. These proinflammatory features may be related to its ability to degrade mucus, leading to breakdown of the gut barrier and increased exposure of resident immune cells to microbial antigens ${ }^{40}$.

We found lower abundances of Butyricimonas, a butyrateproducing genus, in MS patients. Butyrate is a short-chain fatty acid produced by microbes that induce colonic regulatory $\mathrm{T}$ cells ${ }^{41}$. Reductions in colonic butyrate can disrupt barrier function and promote inflammation. Similar to our findings, reductions in butyrate producers have been noted in numerous autoimmune and inflammatory diseases including inflammatory bowel disease, rheumatoid arthritis and type 1 diabetes ${ }^{5,42,43}$.

We investigated untreated MS patients, to examine the microbiota independent of MS disease-modifying therapy. We found that the genera that were altered in the entire MS cohort (Methanobrevibacter, Akkermansia and Butyricimonas) were also altered in the untreated population, suggesting that these effects are not specifically correlated with therapy. Furthermore, within the untreated MS subset, we observed reductions in genera belonging to the family Coriobacteriaceae, including Collinsella

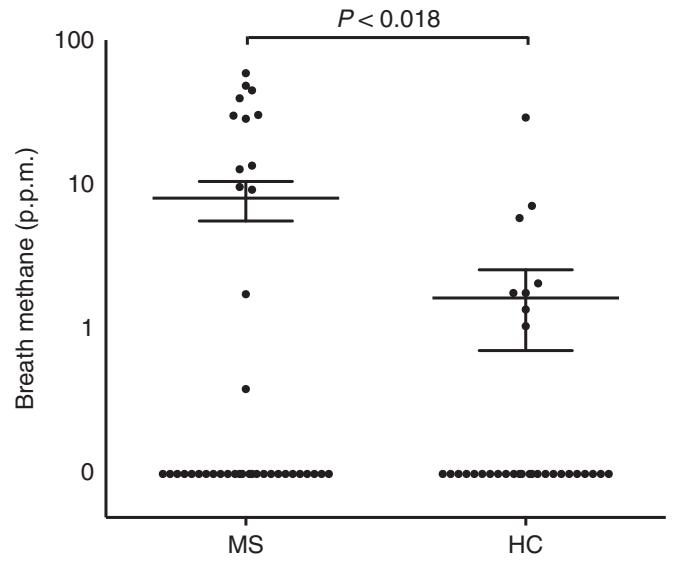

Figure 4 | Measurement of breath methane production in MS patients $(\boldsymbol{n}=\mathbf{4 1})$ and controls $(\boldsymbol{n}=\mathbf{3 2})$. Breath methane measured in each subject is represented on the $y$ axis in parts-per-million on a logarithmic scale. The mean and s.e.m. are shown by the indicated horizontal lines. 28 of $41 \mathrm{MS}$ patients and 24 of 32 controls had no detectable breath methane.

and Slackia. Reductions in Coriobacteriaceae have been reported in relatives of patients with inflammatory bowel disease ${ }^{44}$.

Patients on disease-modifying therapy had increased abundances of the genera Prevotella compared with untreated patients. Although Prevotella has been reported to be increased in rheumatoid arthritis and inflammatory bowel disease ${ }^{5}$, Prevotella has been previously correlated to the intake of high-fibre diets, whose primary substrate, fibre, can drive the generation of the immunoregulatory metabolite butyrate ${ }^{45}$. We found that Prevotella was low in untreated MS, and that treatment with disease-modifying therapy was associated with increased relative abundance of Prevotella. In a smaller cohort of $20 \mathrm{MS}$ patients compared with 40 controls in Japan, the authors detected a decrease in Prevotella ${ }^{11}$ in MS. Given this consistent finding, future studies investigating the role of Prevotella in MS are warranted.

We also observed increases in the genus Sutterella and decreases in Sarcina in MS patients on therapy. Sutterella was found to be increased in healthy controls compared to patients with new-onset Crohn's disease ${ }^{46}$. Sarcina species are reported to be increased in the gut microbiota of autistic patients ${ }^{47}$. Since immunomodulatory treatment in MS was associated with increases in relative abundances of Prevotella and Sutterella and decreases in Sarcina, it is conceivable that treatment may act to normalize a proinflammatory microbiota.

Our finding that some of MS patients have elevated exhaled methane, a surrogate for levels of Methanobrevibacter in the gut, is consistent with our $16 \mathrm{~S}$ rRNA sequencing results; it would be interesting to use this rapid, in vivo test to investigate Methanobrevibacter in larger populations of MS patients. While we did not measure stool abundance of Methanobrevibacter in this second cohort, previous studies have shown that the amount of breath methane strongly correlates with the quantity of M. smithii in the stool ${ }^{26}$. In other anatomical sites, the presence of particular microbial species can be identified based on their metabolic activity, as is routinely done for diagnosing Helicobacter pylori presence with a positive gastric urease test. Future studies employing simultaneous collection of breath, faecal and blood samples in MS patients will address the potential role of breath methane as a biomarker in MS.

The gut microbiota are known to modulate host immune gene expression either by direct contact with cell wall components, or by secretion of factors that can signal through host receptors 
or through epigenetic modifications that may alter methylation or acetylation of transcriptional promoters ${ }^{48}$. Microbial colonization by a single organism or by groups of organisms into the gut of germ-free mice can modulate the expression of innate and adaptive immune genes as early as 4 days after microbial inoculation in varying cellular compartments ${ }^{48}$. In a study of inflammatory bowel disease in humans, associations between microbes and host gene expression were found in innate and adaptive immune pathways ${ }^{49}$.

We examined relationships between microbial abundance and immune genes implicated in MS pathogenesis. Consistent with the inflammatory properties of Methanobrevibacter and Akkermansia $32-34,36,45-46$, we found positive correlations with these organisms and gene expression in T cells and monocytes involved in key pathways previously implicated in MS pathogenesis, including increased expression of the MAPK family in monocytes (MAPK1 and MAPK14), genes directly involved in both the initiation phase of innate immunity and activating adaptive immunity ${ }^{50}$. In T cells, Methanobrevibacter and Akkermansia positively correlated with TRAF5, a known regulator of T-cell activation and known to be overexpressed in MS, as well as STAT5B, whose expression is indispensible for the encephalitogenicity of autoreactive $\mathrm{CD} 4^{+} \mathrm{T}$ cells in $\mathrm{EAE}^{50,51}$. Methanobrevibacter or Akkermansia negatively correlated with TNFAIP3, previously shown to have reduced expression in studies of the MS transcriptome ${ }^{21,50}$. In the case of Akkermansia, these relationships were even stronger among untreated MS patients alone. Butyricimonas had negative correlations with genes known to be increased in MS among T cells and monocytes, suggesting that reduction in Butyricimonas is associated with increased proinflammatory gene expression, however, directionality cannot be determined from this study. These correlations were observed among all subjects and in untreated MS patients, but not in controls alone, suggesting that the relationship between microbial abundance and gene expression was MS-specific, but not solely driven by differences between controls and MS patients. Although we could detect correlation, we cannot determine the causal direction or conclude whether the microbes drive immunological changes, or whether the disease or altered immunity drives changes in the microbiota.

While our investigation of the gut microbiota in MS provides initial insights into understanding the potential role for the microbiome in this disease, our study has certain limitations. First, we cannot assign a direct cause to the associations we describe in the gut microbiome and the MS immunophenotype. The altered microbes may play a role in disease-related immunological changes, or MS-related changes in physiology may drive microbial alterations. Methanobrevibacter, for example, is recovered more frequently from individuals with constipationvariant irritable bowel disease ${ }^{52}$; while we excluded patients with irritable bowel disease from our study, it is possible, for example, that subtle changes in gut motility or in the enteric nervous system in MS patients or co-existing constipation may produce conditions favourable for the growth of this microbe. Second, our findings could be influenced by cohort-specific confounders. To minimize the effects of confounders, we used strict exclusion criteria eliminating the potential influence of antibiotics, pregnancy or other autoimmune or gastrointestinal conditions. Medications taken before the window of our study-such as prior courses of steroids-may also influence gut microbial populations. Furthermore, MS patients often consume alternative diets that might favour the growth of particular microbial niches ${ }^{53}$. Although we did not find any large differences in dietary intake in our cohort, more sensitive assays may reveal dietary variation that may be responsible for the observed changes in the gut microbiome in our disease population. Although the methodology used to collect stool samples was relatively uniform, variability in time of sample collection and interval of previous dietary intake may have also contributed to changes in microbes recovered. While age, gender and BMI have been previously shown to drive changes in the microbiome, our multi-factorial model suggests that these factors did not confound the observed microbial differences between the groups. Although we did not find a relationship between changes in the gut microbiome and clinical parameters such as disease duration or disability, most of our patients had low levels of disability (average EDSS 1.2). Future studies with larger cohorts and longitudinal collection of samples will be required to investigate these clinical associations, including subjects with progressive forms of the disease. Third, we analysed the microbiota using primers targeting the $16 \mathrm{~S}$ rRNA gene. While this is useful for providing taxonomic information, shotgun metagenomic, metatranscriptomic and metabolomic profiling of faecal samples may reveal changes in the abundance of microbial genes, their expression or the presence of microbial metabolites that would complement phylogenic and taxonomic data. Finally, our study is limited by the fact that we collected samples after disease onset. It is possible that critical changes in the gut microbiome in MS may occur in early or preclinical stages of disease. For example, in type 1 diabetes, changes in the gut microbiome were apparent before the onset of illness in high-risk individuals with the concomitant appearance of anti-islet antibodies $^{54}$. Investigation of the microbiota in paediatric or early-onset MS may provide further evidence of associations between the composition of the gut microbiome and MS pathogenesis.

In summary, we have found alterations of the human gut microbiome in MS that correlate with changes in the immune transcriptome and treatment. It is possible that treatment strategies of MS in the future may include therapeutic interventions designed to affect the microbiome such as probiotics, faecal transplantation and delivery of constituents of organisms isolated from the microbiome ${ }^{10}$, although more work is required. In addition, characterization of the gut microbiome in MS may provide biomarkers for assessing disease activity and could theoretically be an avenue to prevent MS in young at-risk populations.

\section{Methods}

Study population. Relapsing-remitting MS patients were recruited from the Partners MS Center at Brigham and Women's Hospital and healthy subjects from the Brigham and Women's Hospital PhenoGenetic project (http://dejager_lab. bwh.harvard.edu/?page_id=2317). Untreated patients were treatment naive or with no steroid treatment in the previous month, no beta-interferon/glatiramer acetate treatment in the previous 3 months, and no other treatments over the prior 6 months. None of the MS patients had an active relapse at the time of sampling. Patients with a history of using other immunosuppressive medications including teriflunomide, cyclophosphamide, mitoxantrone, rituximab, intravenous immunoglobulin, daclizumab, basiliximab, azathioprine, methotrexate or mycophenolate mofetil were excluded. Treated patients in the cohort were those who had received beta-interferon or glatiramer acetate for at least 6 months. Exclusion criteria for both MS subjects and healthy control subjects were as follows: no antibiotic use in the prior 6 months; no probiotic use; corticosteroids; history of gastroenteritis; or travel outside of the country in the prior month. No history of irritable bowel syndrome, bowel surgery, inflammatory bowel disease or other autoimmune disease. Pregnancy was also an exclusion criteria. A dietary survey was administered to all subjects before collection of samples (Supplementary Table 3). The protocol was approved by the Partners Human Research Committee and informed consent was obtained from all subjects.

Sample collection. Stool samples were obtained from patients by providing them with stool collection containers. We used a consistent methodology for processing and storage of all samples. Subjects collected a single-sample produced at any time of day with no specific dietary restrictions. Collection containers were then placed in boxes with provided ice packs for immediate shipment to our laboratory via overnight delivery at a maintained temperature of $0{ }^{\circ} \mathrm{C}$. On receipt of samples, they were frozen at $-80^{\circ} \mathrm{C}$ until DNA extraction ${ }^{55}$. Samples were only subjected to a single free-thaw cycle. 
Preparation of DNA and sequencing protocols. For Roche 454 pyrosequencing, DNA was extracted using the PowerSoil DNA Isolation kit (MO BIO Laboratories, Carlsbad, CA, USA) with the Human Microbiome Project modifications to the manufacturer's protocol ${ }^{56}$. DNA quality and yield were evaluated via agarose gel and Qubit fluorometer (Life Technologies Corporation, Carlsbad, CA, USA). The $16 \mathrm{~S}$ rRNA gene libraries were generated by the Center for Metagenomics and Microbiome Research at Baylor College of Medicine, using the V3-V5 (357F/926R) primer in accordance with standard Human Microbiome Project protocols $s^{55}$. The 16S rRNA libraries were sequenced by the Human Genome Sequencing Center at Baylor College of Medicine using a Roche 454 GS FLX + instrument (Roche, Indianapolis, IN) operated with Titanium chemistry.

For Illumina MiSeq 16S rRNA sequencing, methods were adapted from the protocol developed for the NIH-Human Microbiome Project ${ }^{55}$. Briefly, bacterial genomic DNA was extracted using the MO BIO PowerSoil DNA Isolation Kit (MO BIO Laboratories). The $16 \mathrm{~S}$ rRNA V4 region was amplified by PCR and sequenced on the MiSeq platform (Illumina) using the $2 \times 150 \mathrm{bp}$ paired-end protocol. The primers used for amplification contain adaptors for MiSeq sequencing and dualindex barcodes so that the PCR products may be pooled and sequenced directly ${ }^{57}$. Sequencing was performed at the Human Genome Sequencing Center at Baylor College of Medicine.

$16 \mathrm{~S}$ rRNA sequencing data preprocessing. Sequencing of the 105 human stool samples on the Roche 454 GS FLX + instrument generated 1,426,326 total raw reads. Raw reads were processed using the mothur software package (v.1.34.2)14 (ref. 15), which performs demultiplexing and denoising, quality filtering, alignment against the ARB Silva reference database of $16 \mathrm{~S}$ rRNA gene sequences, and clustering into OTUs (at 97\% identity; Supplementary Fig. 1). In total, 4317 OTUs were generated. After filtering OTUs that failed to have a mean number of reads per sample $\geq 1$ in at least one cohort, 426 OTUs were available for further analysis.

Sequencing of the samples on the Illumina MiSeq instrument generated $11,498,168$ total forward raw reads. Preprocessing was performed using the mothur software package. The mothur MiSeq SOP was followed, except we used a custom Python script to perform base quality trimming (sliding window size of $50 \mathrm{nt}$ with average quality score $>35$ ); this modification was employed to improve the quality of sequencing reads used for OTU clustering, since the standard mothur SOP assumes longer sequencing reads than generated by our MiSeq protocol. 10,620 OTUs were generated, with 1,191 OTUs available for further analysis after filtering using the same procedure as described for the Roche 454 data (Supplementary Fig 1)

16S rRNA sequencing data analysis. A measure of alpha diversity, the Shannon entropy ${ }^{58}$ was calculated for the samples, and the nonparametric Wilcoxon rank-sum test ${ }^{59}$ was applied for hypothesis testing. To visualize differences in overall microbial community structure, the unweighted and weighted UniFrac measures were calculated between all pairs of samples, and Principal Coordinates Analysis plots were generated using custom R scripts ${ }^{60}$. Analysis of molecular variance was used for statistical hypothesis testing of differences in overal microbial community structure between cohorts as assessed with the UniFrac measures ${ }^{61}$.

To statistically test for differences in the relative abundances of microbial taxa (phyla or genera) the DESeq2 software package was employed ${ }^{62,63}$. To control for covariates (gender, age and BMI) of interest, we used the multi-factorial model in DESeq2. The DESeq2 multi-factorial model requires categorical values if $>1$ covariate is included, so we discretized age and BMI values. For BMI, we used standard World Health Organization (WHO) categories of normal, overweight, and obese. We reclassified one subject with a BMI of 18.1 as normal, as this is at the borderline of the WHO underweight classification $(<18.5)$ and would have resulted in only one subject in the underweight category. For age, we divided the range of study subjects' ages (27 to 63 years) into four categories, with each category spanning a 9 -year increment. Eight subjects were missing the clinical data. These subjects were excluded from analyses controlling for covariates.

For all statistical testing for $16 \mathrm{~S}$ rRNA data analysis, $P$ values were adjusted for multiple hypothesis testing using the method of Benjamini and Hochberg ${ }^{64}$.

To more accurately identify the microorganisms present in samples and their phylogenetic relationships to known species, the pplacer software package was used to perform phylogenetic placement ${ }^{16}$. Pplacer uses a likelihood-based methodology to place short sequencing reads of $16 \mathrm{~S}$ rRNA amplicons on a reference tree, and also generates taxonomic classifications of the short sequencing reads using a least common ancestor-based algorithm. The reference tree required for phylogenetic placement was generated using full-length or near full-length $(>1,200 \mathrm{nt}) 16 \mathrm{~S}$ rRNA sequences of type strains from the Ribosomal Database Project (RDP) ${ }^{65}$. 9,563 16S rRNA sequences were downloaded from RDP-11-1, representing 8,719 type strains with 375 sequences from Archaea and 9,188 sequences from Bacteria strains. Filtering was performed using custom Python scripts with the following criteria: (1) only one sequence per species (if the species had more than one sequence, then the longest sequence was chosen); (2) non-environmental species (using key words); (3) no sequences with unclear taxonomic lineage information; (4) no redundant sequences. 7,890 16S rRNA sequences were retained for constructing the phylogenetic tree. Multiple sequence alignment was performed using Muscle ${ }^{66}$. Per the pplacer manual (http://matsen.github.io/pplacer/ generated_rst/pplacer.html), the $16 \mathrm{~S}$ rRNA reference tree was constructed using FastTree with parameter '-nt -gtr' (ref. 67). FastTree infers phylogenetic trees using an approximate maximum likelihood-based approach, and generates local support values to estimate the reliability of each split in the tree by using the Shimodaira-Hasegawa test on the three alternate topologies (NNIs) around that split, counting the fraction of 1,000 resamples that support a split over the two potential NNIs around that node. Local support values of $>0.95$ are considered to strongly support splits, and those $>0.70$ are considered to moderately support splits. To generate the phylogenetic placement figures in the main manuscript (Supplementary Figs 9 and 10), representative sequences for each species within the genus of interest were chosen (the most abundant unique sequence assigned to that species by pplacer).

Methane breath testing. A separate cohort of subjects was utilized for methane breath testing. The inclusion and exclusion criteria used to recruit patients was identical to that described above. Breath samples were collected from subjects after an overnight fast. All subjects brushed their teeth following the 8-hour fast then produced end-expiratory breath samples into provided collection tubes. Collection tubes were sent to Commonwealth Labs (Salem, MA) where gas chromatography was performed to assess the presence of exhaled methane as described previously ${ }^{26}$

Immunologic assays. The Methanobrevibacter smithii type strain (ATCC 35061) was cultured at the University of Massachusetts using methods previously described $^{68}$. Assesment of antibodies against $M$. Smithii was performed by ELISA. Lysates of Methanobrevibacter were prepared from sonicates of Methanobrevibacter cultures. PBMCs were isolated as previously described ${ }^{69}$ PBMCs were stimulated with Methanobrevibacter smithii, tetanus toxoid or anti-CD3/CD28 in 15 healthy donors and 14 MS patients. Methanobrevibacter smithii was cultured in a ratio of 1 bacteria to $1,10,100$ or 1,000 PBMCs. Thymidine incorporation was measured 4 days following stimulation. Cytokine production was measured by Luminex assay (Miltenyi Biotec). Monocytes and T cells were sorted from PBMCs from 20 MS patients and 20 healthy controls (see below) using a Miltenyi Biotec (Alburn, CA) selection kit. Total RNA was extracted using Norgen RNA purification kits (Norgen Biotek Corp., norgenbiotek.com). NanoString expression of immune genes was detected by NanoString array (nCounter, Gene expression code set, Human Immunology kit) as previously described ${ }^{69}$.

Statistical analyses of gene expression and microbiome associations. T cells and monocytes were sorted from PBMCs from 20 MS patients and 20 healthy subjects. These participants were chosen from the larger cohort by selecting $10 \mathrm{MS}$ patients with the highest Methanobrevibacter relative abundance, 10 MS patients with the lowest Methanobrevibacter relative abundance, 10 healthy subjects with the highest Methanobrevibacter relative abundance and 10 healthy subjects with the lowest Methanobrevibacter relative abundance. Of these samples, $\mathrm{T}$ cells and monocytes from two MS patients and two healthy subjects either had low viability of low recovery of RNA thus these samples were excluded from further analysis. Count data were normalized using the nSolver Analysis software (Nanostring), significant differences were detected by $t$-test and $P$ values were adjusted for multiple comparisons using the Benjamini-Hochberg correction to assess significance at the 0.05 level.

Pathway annotations were conducted using the Ingenuity Pathway Analysis (Qiagen) and canonical pathways that had an activation score $>1.5$ or $<-1.5$ in either T cells or monocytes were reported. Spearman's correlations between microbial abundance and immune gene expression was calculated in the $\mathrm{R}$ statistical framework ${ }^{70}$ using the cor.test in the stats package and plotted using the ellipse package ${ }^{71}$

Data availability. The high-throughput sequence data have been deposited in the National Center for Biotechnology Information (NCBI) BioProject database with project number PRJNA321051. The data from the 16S rRNA sequencing on the MiSeq and 454 platforms have been deposited in the NCBI Sequence Read Archive, and linked to the mentioned BioProject number. T-cell and monocyte gene expression data obtained using the Nanostring immunology panel have been deposited in the NCBI Gene Expression Omnibus (GEO) database under accession number GSE81279. The authors declare that all other data supporting the findings of this study are available within the article and its Supplementary Information files, or from the corresponding author on request.

\section{References}

1. Lozupone, C. A., Stombaugh, J. I., Gordon, J. I., Jansson, J. K. \& Knight, R. Diversity, stability and resilience of the human gut microbiota. Nature $\mathbf{4 8 9}$, 220-230 (2012).

2. McDermott, A. J. \& Huffnagle, G. B. The microbiome and regulation of mucosal immunity. Immunology 142, 24-31 (2014).

3. Kostic, A. D., Xavier, R. J. \& Gevers, D. The microbiome in inflammatory bowel disease: current status and the future ahead. Gastroenterology 146, 1489-1499 (2014). 
4. Alkanani, A. K. et al. Alterations in intestinal microbiota correlate with susceptibility to type 1 diabetes. Diabetes 64, 3510-3520 (2015).

5. Scher, J. U. et al. Expansion of intestinal Prevotella copri correlates with enhanced susceptibility to arthritis. Elife 2, e01202 (2013).

6. Berer, K. et al. Commensal microbiota and myelin autoantigen cooperate to trigger autoimmune demyelination. Nature 479, 538-541 (2011).

7. Ivanov, II et al. Induction of intestinal Th17 cells by segmented filamentous bacteria. Cell 139, 485-498 (2009).

8. Lee, Y. K., Menezes, J. S., Umesaki, Y. \& Mazmanian, S. K. Proinflammatory T-Cell responses to gut microbiota promote experimental autoimmune encephalomyelitis. Proc. Natl. Acad. Sci. USA 108(Suppl 1): 4615-4622 (2011).

9. Round, J. L. \& Mazmanian, S. K. Inducible Foxp3+ regulatory T-cell development by a commensal bacterium of the intestinal microbiota. Proc. Natl. Acad. Sci. USA 107, 12204-12209 (2010).

10. Ochoa-Reparaz, J. et al. A polysaccharide from the human commensal Bacteroides fragilis protects against CNS demyelinating disease. Mucosal Immunol. 3, 487-495 (2010).

11. Miyake, S. et al. Dysbiosis in the gut microbiota of patients with multiple sclerosis, with a striking depletion of species belonging to clostridia XIVa and IV clusters. PLoS ONE 10, e0137429 (2015).

12. Machiels, K. et al. A decrease of the butyrate-producing species Roseburia hominis and Faecalibacterium prausnitzii defines dysbiosis in patients with ulcerative colitis. Gut 63, 1275-1283 (2014).

13. Varrin-Doyer, M. et al. Aquaporin 4-specific T cells in neuromyelitis optica exhibit a Th17 bias and recognize Clostridium ABC transporter. Ann. Neurol. 72, 53-64 (2012).

14. Jacobs, B. C. et al. Campylobacter jejuni infections and anti-GM1 antibodies in Guillain-Barre syndrome. Ann. Neurol. 40, 181-187 (1996).

15. Schloss, P. D. et al. Introducing mothur: open-source, platform-independent, community-supported software for describing and comparing microbial communities. Appl. Environ. Microbiol. 75, 7537-7541 (2009).

16. Matsen, F. A., Kodner, R. B. \& Armbrust, E. V. pplacer: linear time maximum-likelihood and Bayesian phylogenetic placement of sequences onto a fixed reference tree. BMC Bioinformatics 11, 538 (2010).

17. Bang, C., Weidenbach, K., Gutsmann, T., Heine, H. \& Schmitz, R. A. The intestinal archaea Methanosphaera stadtmanae and Methanobrevibacter smithii activate human dendritic cells. PLoS ONE 9, e99411 (2014).

18. Cao, Y. et al. Functional inflammatory profiles distinguish myelin-reactive T cells from patients with multiple sclerosis. Sci. Transl. Med. 7, 287ra274 (2015).

19. Schneider, A. et al. In active relapsing-remitting multiple sclerosis, effector $\mathrm{T}$ Cell Res.istance to adaptive T(regs) involves IL-6-mediated signaling. Sci. Transl. Med. 5, 170ra115 (2013).

20. Miterski, B. et al. Inhibitors in the NFkappaB cascade comprise prime candidate genes predisposing to multiple sclerosis, especially in selected combinations. Genes Immun. 3, 211-219 (2002).

21. Gilli, F. et al. Learning from nature: pregnancy changes the expression of inflammation-related genes in patients with multiple sclerosis. PLOS ONE 5, e8962 (2010).

22. Miranda-Hernandez, S. \& Baxter, A. G. Role of toll-like receptors in multiple sclerosis. Am. J. Clin. Exp. Immunol. 2, 75-93 (2013).

23. Fernandez-Paredes, L., de Diego, R. P., de Andres, C. \& Sanchez-Ramon, S. Close encounters of the first kind: innate sensors and multiple sclerosis. Mol. Neurobiol. doi:10.1007/s12035-015-9665-5 (2016).

24. Blais Lecours, P. et al. Immunogenic properties of archaeal species found in bioaerosols. PLoS ONE 6, e23326 (2011).

25. McKay, L. F., Eastwood, M. A. \& Brydon, W. G. Methane excretion in man--a study of breath, flatus, and faeces. Gut 26, 69-74 (1985).

26. Pimentel, M. et al. Methane production during lactulose breath test is associated with gastrointestinal disease presentation. Dig. Dis. Sci. 48, 86-92 (2003).

27. Haberman, Y. et al. Pediatric Crohn disease patients exhibit specific ileal transcriptome and microbiome signature. J. Clin. Invest. 124, 3617-3633 (2014).

28. Dunne, J. L. et al. The intestinal microbiome in type 1 diabetes. Clin. Exp. Immunol. 177, 30-37 (2014).

29. Oksenberg, J. R., Baranzini, S. E., Sawcer, S. \& Hauser, S. L. The genetics of multiple sclerosis: SNPs to pathways to pathogenesis. Nat. Rev. Genet. 9, 516-526 (2008).

30. Verma, R., Verma, A. K., Ahuja, V. \& Paul, J. Real-time analysis of mucosal flora in patients with inflammatory bowel disease in India. J. Clin. Microbiol. 48, 4279-4282 (2010).

31. Yamabe, K. et al. Distribution of Archaea in Japanese patients with periodontitis and humoral immune response to the components. FEMS Microbiol. Lett. 287, 69-75 (2008).

32. Krishnan, L., Sad, S., Patel, G. B. \& Sprott, G. D. The potent adjuvant activity of archaeosomes correlates to the recruitment and activation of macrophages and dendritic cells in vivo. J. Immunol. 166, 1885-1893 (2001).
33. Samuel, B. S. et al. Genomic and metabolic adaptations of Methanobrevibacter smithii to the human gut. Proc. Natl. Acad. Sci. USA 104, 10643-10648 (2007).

34. Mbakwa, C. A. et al. Gut colonization with Methanobrevibacter smithii is associated with childhood weight development. Obesity (Silver Spring) 23, 2508-2516 (2015).

35. Tremlett, H. et al. Gut microbiota composition and relapse risk in pediatric MS: A pilot study. J. Neurol. Sci. 363, 153-157 (2016).

36. Cantarel, B. L. et al. Gut microbiota in multiple sclerosis: possible influence of immunomodulators. J. Invest. Med. 63, 729-734 (2015).

37. Scher, J. U. et al. Decreased bacterial diversity characterizes the altered gut microbiota in patients with psoriatic arthritis, resembling dysbiosis in inflammatory bowel disease. Arthritis Rheumatol. 67, 128-139 (2015).

38. Derrien, M., Vaughan, E. E., Plugge, C. M. \& de Vos, W. M. Akkermansia muciniphila gen. nov., sp. nov., a human intestinal mucin-degrading bacterium. Int. J. Syst. Evol. Microbiol. 54, 1469-1476 (2004).

39. Derrien, M. et al. Modulation of mucosal immune response, tolerance, and proliferation in mice colonized by the mucin-degrader Akkermansia muciniphila. Front. Microbiol. 2, 166 (2011).

40. Ganesh, B. P., Klopfleisch, R., Loh, G. \& Blaut, M. Commensal Akkermansia muciniphila exacerbates gut inflammation in Salmonella typhimurium-infected gnotobiotic mice. PLoS ONE 8, e74963 (2013).

41. Furusawa, Y. et al. Commensal microbe-derived butyrate induces the differentiation of colonic regulatory T cells. Nature 504, 446-450 (2013).

42. Wang, W. et al. Increased proportions of Bifidobacterium and the Lactobacillus group and loss of butyrate-producing bacteria in inflammatory bowel disease. J. Clin. Microbiol. 52, 398-406 (2014).

43. de Goffau, M. C. et al. Aberrant gut microbiota composition at the onset of type 1 diabetes in young children. Diabetologia 57, 1569-1577 (2014).

44. Joossens, M. et al. Dysbiosis of the faecal microbiota in patients with Crohn's disease and their unaffected relatives. Gut 60, 631-637 (2011).

45. Wu, G. D. et al. Linking long-term dietary patterns with gut microbial enterotypes. Science 334, 105-108 (2011).

46. Gevers, D. et al. The treatment-naive microbiome in new-onset Crohn's disease. Cell Host Microbe 15, 382-392 (2014).

47. De Angelis, M. et al. Fecal microbiota and metabolome of children with autism and pervasive developmental disorder not otherwise specified. PLOS ONE 8, e76993 (2013).

48. Levy, M., Thaiss, C. A. \& Elinav, E. Metagenomic cross-talk: the regulatory interplay between immunogenomics and the microbiome. Genome Med. 7, 120 (2015).

49. Morgan, X. C. et al. Associations between host gene expression, the mucosal microbiome, and clinical outcome in the pelvic pouch of patients with inflammatory bowel disease. Genome Biol. 16, 67 (2015).

50. Achiron, A., Gurevich, M., Friedman, N., Kaminski, N. \& Mandel, M. Blood transcriptional signatures of multiple sclerosis: unique gene expression of disease activity. Ann. Neurol. 55, 410-417 (2004).

51. Sheng, W. et al. STAT5 programs a distinct subset of GM-CSF-producing T helper cells that is essential for autoimmune neuroinflammation. Cell Res. 24, 1387-1402 (2014).

52. Kim, G. et al. Methanobrevibacter smithii is the predominant methanogen in patients with constipation-predominant IBS and methane on breath. Dig. Dis. Sci. 57, 3213-3218 (2012).

53. Riccio, P., Rossano, R. \& Liuzzi, G. M. May diet and dietary supplements improve the wellness of multiple sclerosis patients? A molecular approach. Autoimmune Dis. 2010, 249842 (2011).

54. Giongo, A. et al. Toward defining the autoimmune microbiome for type 1 diabetes. ISME J. 5, 82-91 (2011).

55. Human Microbiome Project, C. A framework for human microbiome research. Nature 486, 215-221 (2012).

56. Human Microbiome Project, C. Structure, function and diversity of the healthy human microbiome. Nature 486, 207-214 (2012).

57. Caporaso, J. G. et al. Ultra-high-throughput microbial community analysis on the Illumina HiSeq and MiSeq platforms. ISME J. 6, 1621-1624 (2012).

58. Shannon, C. E. W. W. A Mathematical Theory of Communication. Bell Syst. Tech. J. 27, 379-423 (1948).

59. D. F., Bauer Constructing confidence sets using rank statistics. J. Am. Stat. Assoc. 67, 687-690 (1972).

60. Lozupone, C. \& Knight, R. UniFrac: a new phylogenetic method for comparing microbial communities. Appl. Environ. Microbiol. 71, 8228-8235 (2005).

61. Excoffier, L., Smouse, P. E. \& Quattro, J. M. Analysis of molecular variance inferred from metric distances among DNA haplotypes: application to human mitochondrial DNA restriction data. Genetics 131, 479-491 (1992).

62. Love, M. I., Huber, W. \& Anders, S. Moderated estimation of fold change and dispersion for RNA-seq data with DESeq2. Genome Biol. 15, 550 (2014).

63. McMurdie, P.J. \& Holmes, S. Waste not, want not: why rarefying microbiome data is inadmissible. PLoS Comput. Biol. 10 (2014). 
64. Benjamini, Y. \& Hochberg, Y. Controlling the false discovery rate: a practical and powerful approach to multiple testing. J.R. Stat. Soc. B 57, 289-300 (1995).

65. Cole, J. R. et al. Ribosomal Database Project: data and tools for high throughput rRNA analysis. Nucleic Acids Res. 42, D633-D642 (2014).

66. Edgar, R. C. MUSCLE: a multiple sequence alignment method with reduced time and space complexity. BMC Bioinformatics 5, 113 (2004).

67. Price, M. N., Dehal, P. S. \& Arkin, A. P. FastTree: computing large minimum evolution trees with profiles instead of a distance matrix. Mol. Biol. Evol. 26, 1641-1650 (2009).

68. Miller, T. L., Wolin, M. J., Conway de Macario, E. \& Macario, A. J. Isolation of Methanobrevibacter smithii from human feces. Appl. Environ. Microbiol. 43, 227-232 (1982).

69. Mazzola, M. A. et al. Identification of a novel mechanism of action of fingolimod (FTY720) on human effector T cell function through TCF-1 upregulation. J. Neuroinflamm. 12, 245 (2015).

70. R Development Core Team. R: a Language and Environment for Statistical Computing. https://www.R-project.org/ (2015).

71. Murdoch, D. \& Chow, E. D. ellipse: Functions for Drawing Ellipses and Ellipse-Like Confidence Regions. Cran Repository. Version 0.3-8 (2013).

\section{Acknowledgements}

Funding support for this work included grants from the NIH (R01 NS087226 and R21NS087867) from The National Multiple Sclerosis Society, and from The Harvard Digestive Disease Center (Grant P30-DK034854 subcontracted to the Center for Clinical and Translational Metagenomics, Department of Pathology at Brigham and Women's Hospital).

\section{Author contributions}

S.J., R.G., L.C, F.Q., N.L., G.G., L.B. and H.W. designed the study. S.J., R.G., B.G., S.C., F.S., K.M., P.N., P.K., T.C., P.D. and H.W. collected the samples. N.L, L.C, R.Y., B.P., S.J., R.G. and G.G. performed statistical analyses. S.J., R.G., L.C, N.L., G.G., P.D., L.B. and H.W. wrote the manuscript. S.J., R.G., F.G., M.M., S.L., S. T., B.T., J.H. and K.S. performed laboratory experiments.

\section{Additional information}

Supplementary Information accompanies this paper at http://www.nature.com/ naturecommunications

Competing financial interests: The authors declare no competing financial interests.

Reprints and permission information is available online at http://npg.nature.com/ reprintsandpermissions/

How to cite this article: Jangi, S. et al. Alterations of the human gut microbiome in multiple sclerosis. Nat. Commun. 7:12015 doi: 10.1038/ncomms12015 (2016).

\section{(c) (i)}

This work is licensed under a Creative Commons Attribution 4.0 International License. The images or other third party material in this article are included in the article's Creative Commons license, unless indicated otherwise in the credit line; if the material is not included under the Creative Commons license, users will need to obtain permission from the license holder to reproduce the material To view a copy of this license, visit http://creativecommons.org/licenses/by/4.0/ 